Detection and direction-discrimination of diotic and dichotic ramp modulations in amplitude and phase

Caroline Witton, Michael I. G. Simpson, G. Bruce Henning, Adrian Rees, and G. G. R. Green

Citation: The Journal of the Acoustical Society of America 113, 468 (2003); doi: 10.1121/1.1525286

View online: https://doi.org/10.1121/1.1525286

View Table of Contents: https://asa.scitation.org/toc/jas/113/1

Published by the Acoustical Society of America

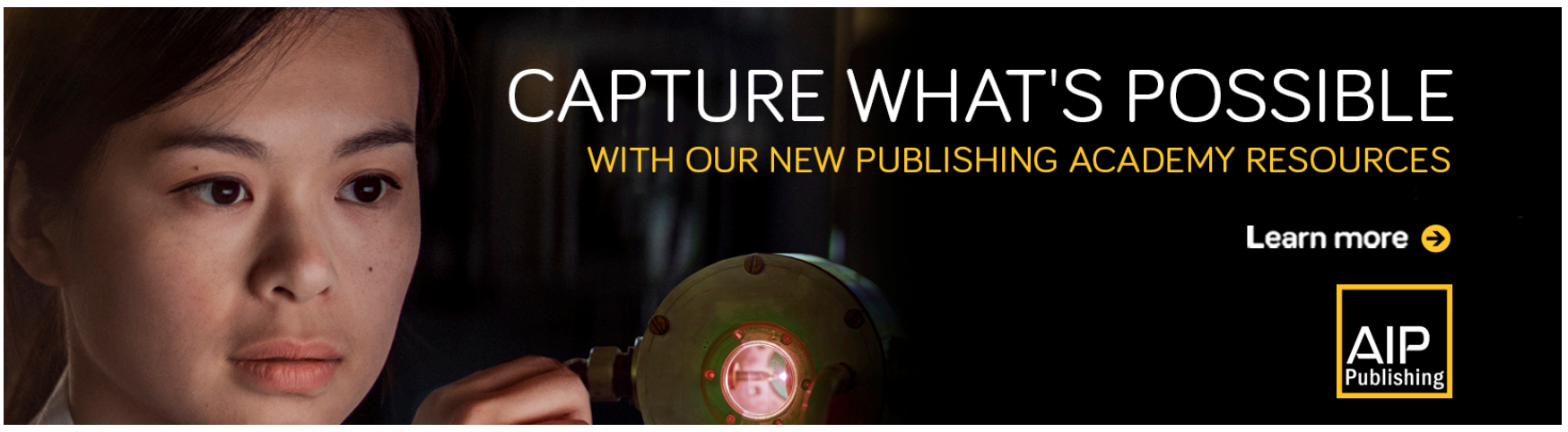




\title{
Detection and direction-discrimination of diotic and dichotic ramp modulations in amplitude and phase
}

\author{
Caroline Witton ${ }^{\text {a) }}$ \\ Neurosciences Research Institute (Behavioural and Cognitive Sciences Research Group), Aston University, \\ Aston Triangle, Birmingham B4 7ET, United Kingdom \\ Michael I. G. Simpson \\ Department of Physiological Sciences, The Medical School, University of Newcastle upon Tyne, \\ Framlington Place, Newcastle upon Tyne NE2 4HH, United Kingdom \\ G. Bruce Henning \\ SRU, Department of Experimental Psychology, University of Oxford, South Parks Road, Oxford OX1 3UD, \\ United Kingdom
}

Adrian Rees and G. G. R. Green

Department of Physiological Sciences, The Medical School, University of Newcastle upon Tyne, Framlington Place, Newcastle upon Tyne NE2 4HH, United Kingdom

(Received 11 July 2001; revised 3 October 2002; accepted 4 October 2002)

\begin{abstract}
When the source of a tone moves with respect to a listener's ears, dichotic (or interaural) phase and amplitude modulations (PM and AM) are produced. Two experiments investigated the psychophysical characteristics of dichotic linear ramp modulations in phase and amplitude, and compared them with the psychophysics of diotic PM and AM. In experiment 1, subjects were substantially more sensitive to dichotic PM than diotic PM, but AM sensitivity was equivalent in the dichotic and diotic conditions. Thresholds for discriminating modulation direction were smaller than detection thresholds for dichotic AM, and both diotic AM and PM. Dichotic PM discrimination thresholds were similar to detection thresholds. In experiment 2, the effects of ramp duration were examined. Sensitivity to dichotic AM and PM, and diotic AM increased as duration was increased from $20 \mathrm{~ms}$ to $200 \mathrm{~ms}$. The functions relating sensitivity to ramp duration differed across the stimuli; sensitivity to dichotic PM increased more rapidly than sensitivity to dichotic or diotic AM. This was also reflected in shorter time-constants and minimum integration times for dichotic PM detection. These findings support the hypothesis that the analysis of dichotic PM and AM rely on separate mechanisms. (C) 2003 Acoustical Society of America. [DOI: 10.1121/1.1525286]
\end{abstract}

PACS numbers: 43.66.Pn [LRB]

\section{INTRODUCTION}

Interaural differences in phase and amplitude permit a listener to determine the horizontal bearing of the source of a tone (Rayleigh, 1907). Moreover, when the source of a continuous tone moves in the horizontal plane with respect to a listener (or the listener's ears move with respect to the tone's source, as when the head is turned), dichotic modulations in the phase and amplitude of the sound are produced. In this paper, we report studies of dichotic phase and amplitude modulation (PM and AM) as cues to motion.

Sinusoidal modulations of dichotic phase can be produced by presenting sinusoidally frequency-modulated (FM) signals to each ear and introducing a phase-delay between the modulations at each ear. Despite the physical similarity between FM and dichotic PM, detection thresholds for sinusoidal dichotic PM are an order of magnitude smaller than monaural or diotic thresholds for sinusoidal FM, when the modulation rate is $1 \mathrm{~Hz}$ (Green et al., 1976; Henning and Zwicker, 1984; Zwicker and Henning, 1985; Witton et al., 2000). This dichotic advantage persists (with decreasing

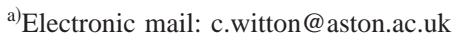

magnitude) up to modulation rates of $40-60 \mathrm{~Hz}$ (Green et al., 1976; Witton et al., 2000), and is not affected by several tens of decibels of fixed interaural level difference (Witton et al., 2000). In other words, at low modulation rates, subjects can make use of the interaural phase-delay present in the dichotic PM stimulus, enabling detection to occur at smaller modulation depths than the detection of monaural or diotic FM. Henning and Zwicker (1984) noted the absence of such an advantage for detecting dichotic over diotic AM.

It has been suggested that the responses of human listeners to moving tone stimuli cued by dichotic PM are sluggish or slow. This suggestion is based principally on observations that thresholds for discriminating modulation of interaural temporal differences (ITD) from FM of a low-pass noise deteriorate rapidly with increasing modulation rate (Grantham and Wightman, 1978). Further evidence for sluggishness was provided by measurements of minimum audible movement angle (MAMA) - the azimuth through which a sound source is required to move for a listener to determine that it is moving or to discriminate the moving source from a stationary sound. Perrott and Musicant (1977) showed that the MAMA for a free-field moving sound increases with increasing sound-source velocity. This finding 
was later confirmed for simulated sound movement (generated by varying the output levels of two loudspeakers), where MAMA increased sharply as stimulus duration was decreased below 100-150 ms (Grantham, 1986), indicating a long time constant or, equivalently, a sluggish system. Chandler and Grantham (1992) again showed that MAMA increased with increasing velocity and calculated a minimum integration time of $336 \mathrm{~ms}$ for dynamic spatial resolution of a $500-\mathrm{Hz}$ tone.

A common problem in sound movement research is that attempts to identify the contributions of velocity, distance, and duration of movement are confounded by their interrelation and it can be difficult to determine which is the controlling variable in an experiment. However, taken with the finding that velocity discrimination thresholds deteriorate with increasing reference velocity, Grantham's data have been interpreted to mean that subjects are relatively insensitive to changes in the velocity of sound movement, and, further, that movement sensitivity is specifically impaired at high movement velocities. Recently, Carlile and Best (2002), using broadband stimuli presented in virtual auditory space, have suggested that the auditory system actually can discern movement velocity, but that velocity discrimination performance is greatly improved by the use of displacement cues in addition to velocity cues.

Grantham $(1982,1984)$ provided evidence that dynamic ITDs are processed differently from dynamic interaural intensity differences (IIDs), when he showed that thresholds for detecting fluctuating IIDs increase less than thresholds for detecting fluctuating ITDs as the rate of fluctuation is increased. There is, therefore, evidence for some difference between the temporal aspects of the mechanisms by which dichotic phase and amplitude cues are processed. It is possible that such differences could have affected the results of previous studies of sound movement perception that have used stimuli composed of different cues.

In this study, we sought to extend the findings of Witton et al. (2000) by examining the psychophysics of dichotic linear ramp modulations similar to those which occur during the movements used in previous studies (e.g., Perrott and Musicant, 1977; Grantham, 1986; Chandler and Grantham, 1992). These ramp modulation stimuli give a percept of smooth unidirectional horizontal movement. We first determined detection thresholds for linear dichotic PM ramps, and for linear diotic PM ramps where no interaural delay was present. Then, to investigate sensitivity to direction (rather than the simple presence of a modulation) we measured thresholds for discriminating between leftwards and rightwards movement. Analogous measurements were also made for dichotic and diotic amplitude modulations, allowing us to test Henning and Zwicker's finding that no dichotic advantage occurs for AM stimuli (Henning and Zwicker, 1984). In a second experiment, we investigated how sensitivity to linear ramps in dichotic PM and AM was affected by restricting the duration of modulation (and hence the extent and duration of any perceived motion). This experiment was designed to allow the comparison of thresholds for isolated dichotic PM and dichotic AM with the data from Grantham (1986) and Chandler and Grantham (1992), and to allow us, like

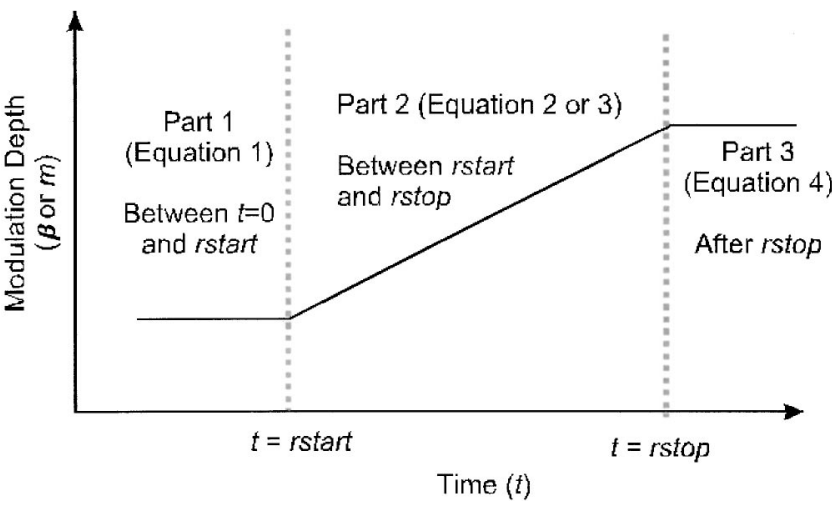

FIG. 1. Sketch of the ramp modulation as a function of time. The stimulus is divided into three parts; the modulation itself begins at time rstart and ends at time rstop. See text for details.

Grantham, to calculate time constants for detection of these modulations.

\section{EXPERIMENT 1. DETECTION AND DISCRIMINATION THRESHOLDS FOR 1-s RAMP MODULATION STIMULI}

\section{A. Methods}

\section{Subjects}

Five trained subjects took part in experiments to measure detection and direction-discrimination thresholds for dichotic ramp modulations of phase and then of amplitude. Three of the subjects also took part in threshold measurements for diotic phase and amplitude ramps. The subjects were aged between 20 and 45 years of age, one was female, and none had any known hearing loss or neurological disorder. All of the subjects underwent a period of at least $5 \mathrm{~h}$ training before data collection.

\section{Stimuli}

The signals were generated using Tucker-Davis Technology equipment (TDT System II). The required waveforms were created digitally and scaled to fill the dynamic range of two independent 16-bit digital-to-analog converters. The sampling rate was $40 \mathrm{kHz}$ and the signals for each ear were low-pass filtered at $12 \mathrm{kHz}$, separately attenuated, and used to drive calibrated Sennheisser HD40 earphones working in phase.

The modulations were linear ramps in phase or amplitude, imposed on a $500-\mathrm{Hz}$ pure tone and presented at $55 \mathrm{~dB}$ SL. The stimuli had a total duration of $1000 \mathrm{~ms}$ and are best described by subdividing them into three parts, each of which can be defined by a single equation. This subdivision is illustrated in Fig. 1. For the first part of the signal, $s(t)$, a pure unmodulated tone of frequency $f$ (equal to $500 \mathrm{~Hz}$ in the present study), of amplitude $A$, occurs for $20 \mathrm{~ms}$ and is given by

$$
s(t)=A \sin (2 \pi f t) \quad(0 \leqslant t \leqslant 0.02) .
$$

During this 20-ms unmodulated portion of the stimulus, the tone is gated on with a half-Gaussian envelope. The second, modulated portion of the stimulus (duration $960 \mathrm{~ms}$ ) can be an increase (as illustrated in Fig. 1) or a decrease in phase or 
amplitude, and is defined differently according to the parameter being modulated. The signal associated with a linear phase modulation (PM) is described as

$s(t)=A \sin \left(2 \pi f t+\frac{\beta(t-r s t a r t)}{r s t o p-r s t a r t}\right) \quad(r s t a r t \leqslant t \leqslant r s t o p)$,

where the modulation index (i.e., the maximum excursion of the ramp, equivalent to modulation depth expressed in radians, but conventionally reported without units) is denoted $\beta$, and the terms rstart and rstop denote the times at which the ramp modulation begins and ends; see Fig. 1 for details. As the phase of the signal changes linearly, there is an accompanying frequency change, in the form of a square pulse. The magnitude of this frequency change is determined by the rate at which the phase is modulated.

When linear AM is applied, the signal can be described as follows:

$s(t)=A\left(1+\frac{m(t-r \text { start })}{(\text { rstop }- \text { rstart })}\right) \sin (2 \pi f t) \quad($ rstar $t \leqslant t \leqslant r$ stop $)$,

where the symbol $m$ denotes the modulation index (for AM, this is equivalent to the modulation depth as a proportion of the total amplitude of the signal).

The third and final portion of the stimulus, after termination of the modulation, is described by Eq. (4) if PM was applied to the signal, or by Eq. (5) if AM was applied:

$$
\begin{aligned}
& s(t)=A \sin (2 \pi f t+\beta) \quad(\text { rstop } \leqslant t \leqslant r s t o p+0.02), \\
& s(t)=A(1+m) \sin (2 \pi f t) \quad(\text { rstop } \leqslant t \leqslant \text { rstop }+0.02) .
\end{aligned}
$$

The duration of the third part of the stimulus was $20 \mathrm{~ms}$ during which the sound was gated off with a half-Gaussian envelope.

The modulation depths in the second and third parts of the stimulus [Eqs. (2)-(5)], $\beta$ and $m$, were positive for an increasing ramp (as in Fig. 1), and negative for a decreasing ramp. Note that for a sinusoidal modulation, the modulation index would normally describe the amount by which the signal is modulated above and below its average. For these stimuli, it represents a unidirectional increase or decrease in the parameter being modulated.

If dichotic modulation was required, the ramps were applied to the subjects' ears with an interaural phase difference such that an increase in the modulated parameter in one ear was matched with a decrease of equal depth in the other ear. If diotic modulation was required, identical signals were presented at the ears. For a positive (upwards) diotic phase or amplitude modulation of a given depth, $D$, the modulation depth of the ramp in each ear was $D$. For a positive (rightwards) dichotic modulation of the same depth, the modulation depth was $+D$ in the right ear and $-D$ in the left ear. Therefore, in this example, the modulation depth, considered at each ear independently ("monaural" depth), was always $D$. When the degree of interaural modulation is considered, however, the diotic and dichotic stimuli differ. For a diotic modulation, the ramps in both ears are identical and there is an interaural phase or amplitude modulation of 0 . Conversely, when ramps are presented in opposite directions (as in the dichotic condition), there is an interaural modulation of $2 D$, i.e., twice the depth of "monaural" modulation. In this paper, all thresholds are presented in terms of the "monaural" depth of modulation at criterion; however, dichotic phase or amplitude thresholds can be converted to threshold depth of interaural phase or amplitude modulation by multiplying by a factor of 2 . $^{1}$

When identical PM is applied to both ears with no interaural phase difference, as in our diotic PM ramps, the resulting stimulus is equivalent to a diotic frequency change. Because frequency change is the derivative of phase change, the frequency modulation associated with the linear phase-ramp takes the form of a square pulse, not a linear frequency ramp. The magnitude of this square frequency pulse is determined by the slope of the phase ramp, and the pulse is of the same duration as the phase ramp. If the duration of a phase ramp of a given depth is increased, its slope-and hence the magnitude of the frequency pulse-decreases. Phase ramp detection thresholds can thus be converted to thresholds for discrimination of frequency pulses in $\mathrm{Hz}(\Delta f)$ as follows:

$$
\Delta f=\frac{\beta}{2 \pi(\text { rstop }- \text { rstart })},
$$

where $\beta$ is the phase ramp depth in modulation index and $($ rstop $-r s t a r t)$ is the ramp duration in seconds (see Fig. 1 for details). Therefore, where appropriate, PM detection thresholds are numerically expressed as frequency changes as well as phase changes.

\section{Psychophysics}

In the detection paradigm, the target interval contained a modulated tone and the other interval contained a pure tone of the same duration. When the modulation was dichotic, the waveforms were presented in such a way that an increase in amplitude or phase occurred at the right ear, and a decrease at the left ear. This resulted in the percept of sound movement towards the right. When the modulation was diotic, increases in phase or amplitude were presented to both ears, in phase, resulting in perception of an increase in intensity, or in a higher pitch. Subjects were simply required to report in which interval, first or second, the modulation had occurred.

In the discrimination paradigm, the target interval was identical to that in the detection paradigm but both intervals contained a modulation. Thus, for dichotic modulations in the target interval, an increase in phase or amplitude was presented to the right ear and a decrease to the left (resulting in a percept of movement to the right). The other interval contained modulations in the opposite direction, i.e., a decrease in phase or amplitude in the right ear, and an increase in the left ear. Thus, in one interval the movement of the intracranial image was to the right, and in the other interval this movement was to the left. Subjects were required to report in which interval they perceived movement to the right. For the diotic condition, in the target interval, an increase in amplitude or phase was presented to both ears and in the other interval, a decrease. Subjects were required to report which interval contained an increase in amplitude or the higher pitch. 


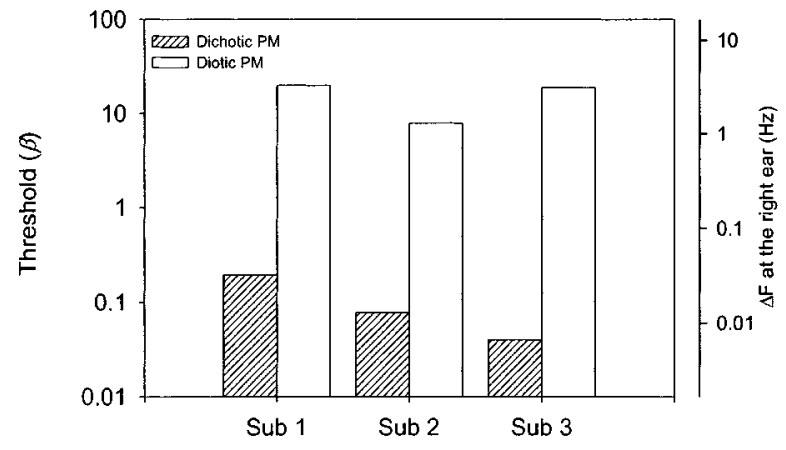

(a) Dichotic and diotic PM detection

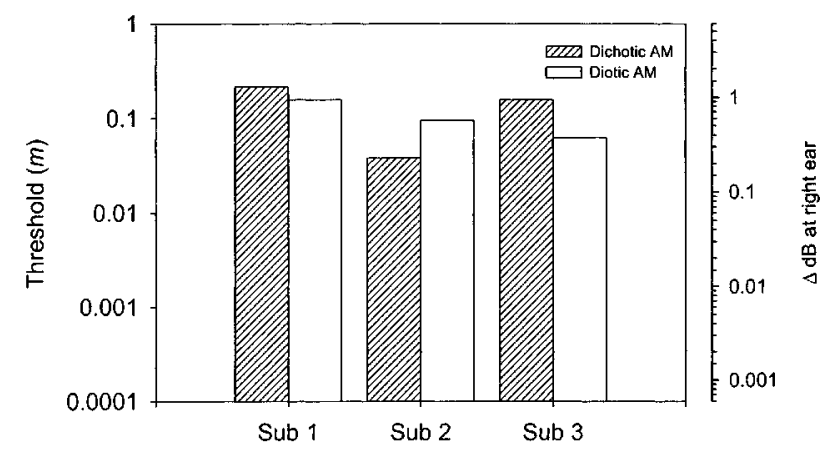

(b) Dichotic and diotic AM detection

FIG. 2. (a) Thresholds for detecting diotic and dichotic PM and (b) thresholds for detecting diotic and dichotic AM. Each pair of bars represents data from a single subject. Note the logarithmic scale on the ordinate.

The discrimination paradigm contained two opposite modulations, whereas the detection paradigm compared a modulation with a pure tone. Thus the extent of modulation, when considered across both intervals, was twice as far in the discrimination paradigm as it was in the detection paradigm. This was true for both diotic and dichotic modulations.

Subjects responded using a set of push buttons, and feedback was provided by lights, which indicated the correct response after the subject had made his or her choice. For each experiment, at least 100 trials were performed at each of 6 stimulus depths, to create a psychometric function. The data were then fitted with a Weibull function (Wichmann and Hill, 2001a, b), allowing the estimation of threshold, which was defined as the stimulus depth corresponding to $75 \%$ correct. All data were collected in an IAC soundproofed room.

\section{B. Results and discussion of experiment 1}

When presented with dichotic ramps in phase or amplitude, all five subjects reported perceiving smooth linear movement of the intracranial image. Subjects reported that the increasing diotic amplitude ramps were perceived as a smooth increase in loudness, and the increasing diotic phase ramps were perceived as having a higher pitch and viceversa for decreasing ramps. For the subject, the diotic PM detection and discrimination tasks were indistinguishable from a standard frequency difference limens task.

\section{Ramp detection}

Figure 2 shows thresholds for detection of dichotic and diotic PM [Fig. 2(a)], and dichotic and diotic AM [Fig. 2(b)] for the three subjects who took part in all conditions. The dichotic performance of the other two subjects was similar to that for the three presented here (see the following section).

The ordinate is shown on a logarithmic axis; depth of phase change is expressed on the left ordinate. Diotic PM detection thresholds (clear columns) are of a similar order of magnitude for all three subjects $($ mean $=15.5[\mathrm{SD}=6.7])$. We can compare diotic PM thresholds in this study with those from Witton et al. (2000) by converting into $\mathrm{Hz}$ [Eq. (6); see Sec. II A for details]. This yields threshold values of 3.3 for subject $1,1.3$ for subject 2 , and 3.1 for subject 3 . Units of monaural frequency change are indicated on the right ordinate of Fig. 2. These values are approximately equivalent to thresholds for detecting 1-Hz FM in our previous study (about 1.5-4 Hz) and are in accordance with other estimates of frequency discrimination threshold for long tone durations (e.g., Henning 1970).

There is some intersubject variability for dichotic PM detection (shaded columns; mean $=0.1$ [s.d.=0.08]), but sensitivity to dichotic PM is greater than to diotic PM for all three subjects [Fig. 2(a)]. Thus, the dichotic advantage for detecting PM, previously described for sinusoidal stimuli (Green et al., 1976; Henning and Zwicker, 1984; Zwicker and Henning, 1985; Witton et al., 2000), was also observed in the data described here. In those previous studies, the amount of interaural phase modulation was identical to the amount of monaural phase modulation, so dichotic advantage could be expressed by simply taking the ratio of the thresholds in each condition. Here, however, the interaural phase modulation in the dichotic condition is twice the phase modulation in each ear. If subjects detect dichotic PM on the basis of the monaural phase modulation, we could simply compare diotic and dichotic PM thresholds, which give 100fold advantages for subjects 1 and 2, and a 470-fold advantage for subject 3 . On the other hand, if the auditory system makes use of the interaural phase modulation, then the measured dichotic PM thresholds, which represent only the phase change at one ear, should be doubled to account for the overall interaural phase modulation in the stimulus. This yields 50-fold advantages for subjects 1 and 2, and a factor of about 236 for subject 3 . Whichever way the advantage is calculated, subjects are clearly substantially more sensitive to PM in the dichotic than the diotic condition as can be seen from the plots in Fig. 2. In addition, the values from this study are larger than those reported by Witton et al., for $1-\mathrm{Hz}$ modulations where advantages of 10-20-fold were obtained. This difference may be because the ramp modulations used here contain much slower modulations than the $1-\mathrm{Hz}$ sinusoidal modulations used by Witton et al. (2000). Green et al. (1976) used modulation rates as low as $0.2 \mathrm{~Hz}$ and also found larger dichotic advantages.

When thresholds for detecting dichotic amplitude ramps are compared with thresholds for diotic amplitude ramps [Fig. 2(b)], a different pattern is observed. Diotic (clear columns) and dichotic (shaded columns) amplitude ramp detection thresholds are similar for all three subjects (diotic AM: 


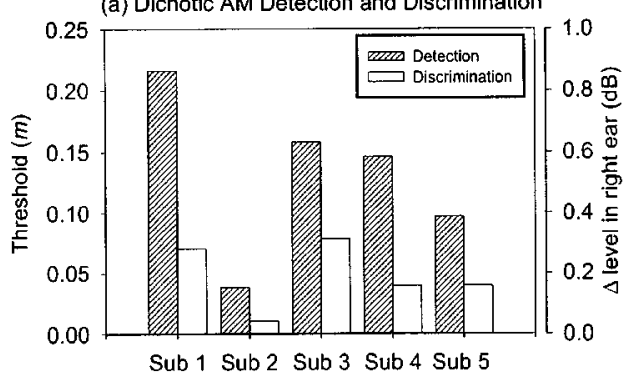

(c) Diotic AM Detection and Discrimination

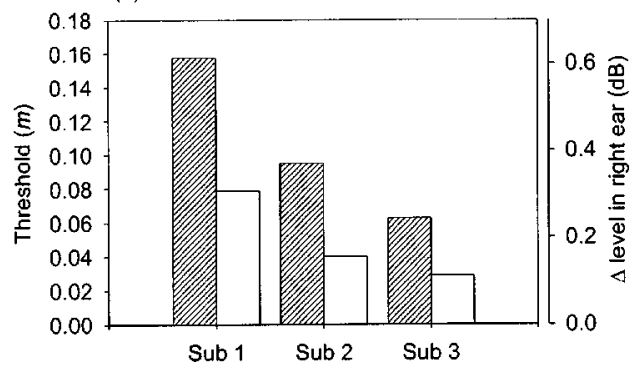

(b) Dichotic PM Detection and Discrimination

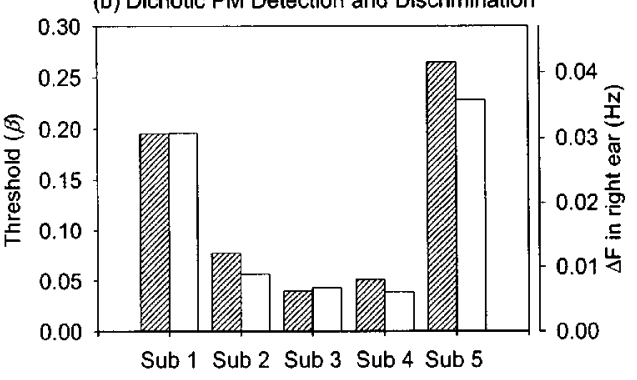

(d) Diotic PM Detection and Discrimination

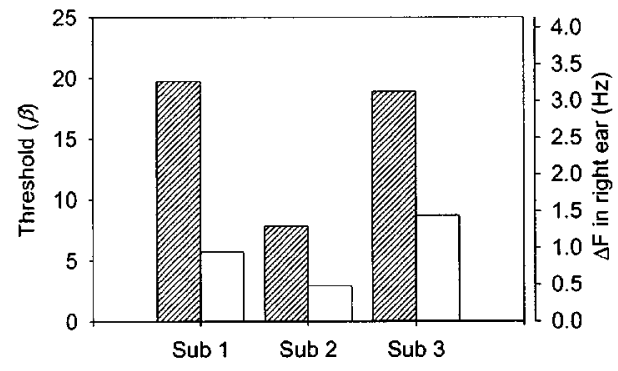

FIG. 3. Detection and directiondiscrimination thresholds for (a) dichotic AM, (b) dichotic PM, (c) diotic AM, and (d) diotic PM ramps. Each pair of bars on each chart represents data from a single subject; shaded bars denote detection thresholds and unfilled bars denote discrimination thresholds. mean $=0.11[$ s.d. $=0.05]$; dichotic AM: mean $=0.14 \quad[$ s.d. $=0.09]$ ). Subjects 1 and 3 have a smaller threshold in the diotic condition, whereas subject 2 has the opposite pattern for thresholds. When a simple ratio of thresholds is taken, the advantages are factors of 0.7 and 0.4 for subjects 1 and 3, respectively, and 2.5 for subject 2 , where a number less than unity denotes a "negative advantage," or a disadvantage. When dichotic AM thresholds are multiplied by 2, to account for the overall interaural amplitude modulation present in the stimulus, the advantages become 0.35 and 0.2 for subjects 1 and 3 , and 1.2 for subject 2. Therefore, although some degree of dichotic advantage was observed for amplitude ramps, it is not consistent across all subjects and the effect is negligible compared to that observed for detection of dichotic and diotic phase ramps.

\section{Ramp discrimination}

Figures 3(a)-(d) show both the modulation detection (shaded columns) and discrimination (clear columns) thresholds for each subject, for dichotic and diotic PM, and dichotic and diotic AM, all on linear axes. Table I shows discrimination thresholds, for each subject, expressed as a percentage of the detection threshold for each modulation type.

TABLE I. Ramp discrimination thresholds, expressed as a percentage of detection threshold for the same ramp modulation, for each subject and each stimulus. The bottom row shows the mean and standard deviation of percentages for each stimulus, across all subjects who collected data in each condition.

\begin{tabular}{ccccc}
\hline \hline Subject no. & Dichotic AM & Dichotic PM & Diotic AM & Diotic PM \\
\hline 1 & $33 \%$ & $100 \%$ & $50 \%$ & $29 \%$ \\
2 & $28 \%$ & $76 \%$ & $42 \%$ & $37 \%$ \\
3 & $49 \%$ & $110 \%$ & $46 \%$ & $46 \%$ \\
4 & $27 \%$ & $76 \%$ & $\ldots$ & $\ldots$ \\
5 & $41 \%$ & $86 \%$ & $\ldots$ & $\ldots$ \\
Mean (sd) & $36 \%(9.3 \%)$ & $90 \%(15 \%)$ & $46 \%(4 \%)$ & $37 \%(8.5 \%)$ \\
\hline \hline
\end{tabular}

In the discrimination paradigm, the maximum extent of modulation across both intervals was twice that in the detection paradigm, since the discrimination intervals contained modulations in opposite directions and of equal depths. If subjects base psychophysical decisions on the overall extent of modulation, rather than on the simple percept of modulation direction, discrimination thresholds could be as little as $50 \%$ of detection thresholds.

Discrimination thresholds for dichotic AM [Fig. 3(a)] are between $27 \%$ and $49 \%$ of those obtained in the detection condition (Table I), suggesting that subjects are at least able to make use of extent-of-modulation information in performing this task. There is also large variability in absolute threshold magnitude, clearly seen in Fig. 3(a). This is in accordance with previous observations of wide intersubject variability in studies of perception of moving sounds (e.g., Grantham, 1986). The fact that some subjects obtained discrimination thresholds substantially lower than $50 \%$ of detection thresholds (e.g., 27\%) is difficult to interpret. Our fitting procedure (Wichmann and Hill, 2001a, b) provides estimates of the standard deviation of the thresholds; obtaining a detection-discrimination ratio as small as 0.27 when the true ratio is 0.5 is somewhat unlikely and may well merit further exploration.

Data for the detection and discrimination of dichotic phase ramps are shown in Fig. 3(b) and in Table I. As for the dichotic AM tasks, the overall extent of modulation in the discrimination paradigm was twice that in the detection paradigm. However, for dichotic PM, subjects' discrimination thresholds were a minimum of $76 \%$ of their detection thresholds, indicating that the information about overall extent of modulation in the discrimination trials did not contribute to thresholds. This observation suggests that there is a major difference between the ways in which subjects detect or discriminate dichotic PM and dichotic AM. As observed for dichotic AM detection and discrimination thresholds, there is a degree of intersubject variability in the absolute magnitude of the dichotic PM detection and discrimination thresholds. 
Data for three subjects' detection and discrimination of diotic ramps in amplitude and phase can be found in Figs. 3(c) and (d), respectively, and in Table I. For amplitude ramps, the ratio of thresholds varies between $42 \%$ and $50 \%$, indicating that, like for dichotic AM, subjects are able to make use of information about the difference between the endpoints of the amplitude modulation in the discrimination paradigm.

In the diotic phase ramp condition, subjects were essentially performing a frequency difference limens task in both the detection and discrimination paradigms (i.e., discriminating between two tones of different pitch). In the discrimination paradigm, the tones were simply twice as far apart in frequency as in the detection paradigm. Discrimination thresholds would therefore still be expected to be approximately $50 \%$ of detection thresholds for diotic phase, even if subjects could not make use of information about the difference between the endpoints of the phase-modulation. Diotic PM discrimination thresholds were indeed found to be $50 \%$, or less than $50 \%$, of detection thresholds, for all three subjects who participated.

In summary, for dichotic AM, and diotic AM and PM, discrimination thresholds are $50 \%$ or less than $50 \%$ of detection thresholds, indicating that subjects are able to make use of information about the overall extent of modulation when discriminating between two opposite modulations. The lack of even a 50\% threshold-decrease in the discrimination paradigm for dichotic PM indicates that subjects are unable to use extent-of-modulation information to perform this task.

\section{Discussion of experiment 1}

Although dichotic ramps in amplitude and phase are perceived similarly (all the subjects reported hearing a smooth, linear movement of the apparent source of the sound from the midline towards the right ear), it is evident from the data described here that there are some significant differences between the detection-discrimination differences for these two dichotic stimuli. The psychophysics of dichotic and diotic AM are similar, but there are clear differences between the psychophysically determined characteristics of dichotic and diotic PM.

\section{The dichotic advantage}

As reported previously (Witton et al., 2000; Henning and Zwicker, 1984; Zwicker and Henning, 1985), it was found that subjects are more sensitive to PM in the dichotic than the diotic condition. This dichotic advantage was greater than previously observed for $1-\mathrm{Hz}$ sinusoidal modulations, perhaps because the modulations in our ramp stimuli were slower and modulated in only one direction over the 1-s stimulus interval. Dichotic PM detection is, thus, not determined simply by the detection of diotic or single-ear modulation.

The lack of a large dichotic advantage for detection of dichotic over diotic AM is in accordance with previous findings (Henning and Zwicker, 1984; Zwicker and Henning, 1985). Subjects do not appear to gain any advantage in detecting dichotic AM from the binaural cues available in the interaural modulation, and threshold appears to be limited by diotic sensitivity to amplitude change rather than by any other measurable factor.

Our diotic PM detection task was essentially a frequency-difference limens task, and this is supported by the similarity of our thresholds with those reported by Henning (1970) and others for frequency discrimination. Dichotic PM detection could (at low modulation rates) be based on the percept of motion, or on some other qualitative perceptual difference, as the modulation depth at threshold is below that at which pitch cues could be used.

The lack of a clear dichotic advantage for AM could suggest that dichotic and diotic AM are processed in the same way; for example, dichotic AM could be detected simply by listening "monaurally" to the AM which occurs at either ear. However, the fact that the perception of the signal is fundamentally different for dichotic and diotic AM suggests that they are indeed sensitive to the interaural changes that occur in the dichotic AM stimulus. This implies that the auditory system does possess a mechanism that can specifically detect dichotic AM, although this may be limited by diotic sensitivity to AM. Our data simply show that subjects are approximately equally sensitive to diotic and dichotic AM.

\section{Detection versus discrimination thresholds}

Dichotic and diotic AM discrimination thresholds were less than $50 \%$ of detection thresholds for the same stimuli. This observation implies that our subjects were making use of information about the difference between the endpoints of the modulations (i.e., overall extent of modulation). This was also the case for the diotic PM tasks, but as noted above subjects were probably basing their decisions on pitch differences rather than modulation.

For the dichotic PM thresholds, discrimination thresholds were on average $90 \%$ of detection thresholds. The data indicate that subjects are not basing their psychophysical judgments on the overall extent of interaural phase modulation across the two intervals, even though they are sensitive to the direction of the modulations, as evidenced by the fact that they are capable of performing the discrimination. The small magnitude of the threshold differences between the detection and discrimination thresholds (especially for subjects 1 and 3) implies that they might be making use of the same qualitative information when performing the detection task as well.

\section{EXPERIMENT 2: THE EFFECT OF RAMP DURATION ON DETECTION THRESHOLDS}

Chandler and Grantham (1992) showed that sensitivity to free-field sounds moving linearly in the horizontal plane decreased sharply when the duration of the movement was restricted below $200 \mathrm{~ms}$, indicating a sluggish system. The results of experiment 1 have suggested that there are some significant differences between the psychophysically defined characteristics of dichotic PM and AM sensitivity. Therefore, in experiment 2, we compared the effects of restricting the duration of the ramp modulation on sensitivity to dichotic $\mathrm{PM}$ and dichotic AM. 


\section{A. Methods}

\section{Subjects}

The subjects were subjects 1 and 3 from experiment 1 , and one additional subject ("subject 6"), and they were all aged between 20 and 45 years. All underwent a training period of at least $5 \mathrm{~h}$ before beginning to collect data.

\section{Stimuli}

Stimuli were dichotic ramp modulations in phase or in amplitude, and diotic phase and amplitude ramps. They were designed exactly as described in Sec. II A, except that the duration of the ramp portion of the stimulus (part 2 in Fig. 1) was varied so that thresholds could be measured for different ramp durations. The duration of the pure tone in the other interval was always the same as the total duration of the modulated tone in the other interval. For example, a $20-\mathrm{ms}$ ramp with 20-ms rise and fall times (a total of $60 \mathrm{~ms}$ ) was always accompanied by a $60-\mathrm{ms}$ pure tone (including $20-\mathrm{ms}$ rise and fall times) in the nontarget interval. The carrier frequency was $500 \mathrm{~Hz}$ and the interstimulus interval was 500 ms.

\section{Psychophysics}

Detection thresholds for each modulation were measured using the same psychophysical procedure as for experiment 1 . Thresholds were obtained for dichotic and diotic PM and $\mathrm{AM}$ at ramp durations between 20 (a 60-ms stimulus when rise and fall portions are added in; see Fig. 1) and 960 ms (a total sound duration of $1 \mathrm{~s}$.).

\section{B. Results of experiment 2}

Data for all three subjects' detection of dichotic and diotic AM and PM at ramp durations between 20 and $960 \mathrm{~ms}$ are shown in Fig. 4.

\section{Effects of ramp duration on detection threshold}

Figure 4(a) shows detection thresholds for dichotic PM and AM as a function of ramp duration for all three subjects. Comparison of the two duration-dependency curves reveals that for both dichotic AM and PM sensitivity is greatest at the longest ramp durations. When considered in terms of modulation index, thresholds for both dichotic PM and dichotic AM are comparable at these longer ramp durations. Thresholds are highest for the shortest ramp duration $(20 \mathrm{~ms})$ and for both modulations they decrease sharply and appear to asymptote with increasing ramp duration. However, the rate of decrease in threshold with ramp duration differs for each modulation type. Dichotic PM detection thresholds decrease more sharply, approaching their minimum value at a duration of about $100 \mathrm{~ms}$ whereas dichotic AM detection thresholds do not approach this plateau until durations are at least 200 ms. This pattern is the same for all three subjects, although the threshold of subject 6 at $20 \mathrm{~ms}$ is comparatively less elevated than for subjects 1 and 3, so the increase in sensitivity with increasing duration is not as pronounced for this listener.
Figures 4(b) and (c) show thresholds for detecting diotic AM and PM, respectively. In Fig. 4(b), dichotic AM detection thresholds from Fig. 4(a) are replotted, and, similarly, in Fig. 4(c) dichotic PM thresholds from Fig. 4(a) are replotted, for the purposes of comparison of thresholds for detecting diotic and dichotic modulations.

In Fig. 4(b), it can be seen that detection thresholds for diotic AM decrease as durations are increased up to about $200 \mathrm{~ms}$, in the same way as for dichotic AM thresholds. Dichotic and diotic AM thresholds have approximately the same duration-dependency, although this similarity is less pronounced for subject 1 between durations of about 50 and $200 \mathrm{~ms}$. Subject 6 has an approximately twofold dichotic advantage at the shortest ramp duration $(20 \mathrm{~ms})$ but this decreases in an irregular way with increasing duration and is reversed at durations above $200 \mathrm{~ms}$. No such dichotic advantage is consistently observed for subjects 1 or 3 , which confirms the lack of advantage observed in experiment 1 .

In Fig. 4(c), it can be seen that detection thresholds for diotic PM, plotted in terms of phase modulation index, increase with increasing ramp duration. This increase is sharpest at the shortest ramp durations, between 20 and $160 \mathrm{~ms}$. The shape of the curves confirms that the slope of the phase change, instead of just the extent, may be important in determining threshold. Phase modulation thresholds can be converted into equivalent $\Delta f$, following Eq. (6) (see also Sec. II A). Figure 5 shows the diotic PM detection threshold data plotted as a function of the frequency change calculated using this method. Plotted in this way the thresholds decrease with increasing duration, like the curves relating thresholds for the other ramp modulations to ramp duration. This pattern of duration dependency is very similar to the many previous accounts of the effects of tone duration on frequency difference limens (e.g., Turnbull, 1944; König, 1957; Henning, 1970; Moore, 1972), illustrating that subjects are effectively performing a frequency discrimination task when detecting diotic phase ramps.

\section{Time constants}

The curves relating thresholds for dichotic PM, frequency pulse detection, and dichotic and diotic AM to duration were fitted with exponential functions, to enable comparison of time constants for the decay of sensitivity. The only exponential function that fit the data to give a significant correlation coefficient $(r)$ was a single exponential with three parameters,

$$
y=y_{0}+a e^{-t / T},
$$

where $t$ and $y$ are the duration and threshold values respectively, $y_{0}$ is the asymptotic threshold value, $a$ is a scalar and $T$ is the time constant. All of the $r$-values from these fits were greater than $0.900(p<0.001)$, except for the dichotic PM data of subject 6 , which could not be significantly fit with a single exponential. Table II shows the time constants calculated using this method for all three subjects, for dichotic $\mathrm{PM}$, dichotic and diotic AM, and frequency pulses.

Time constants for dichotic PM and frequency pulses are 

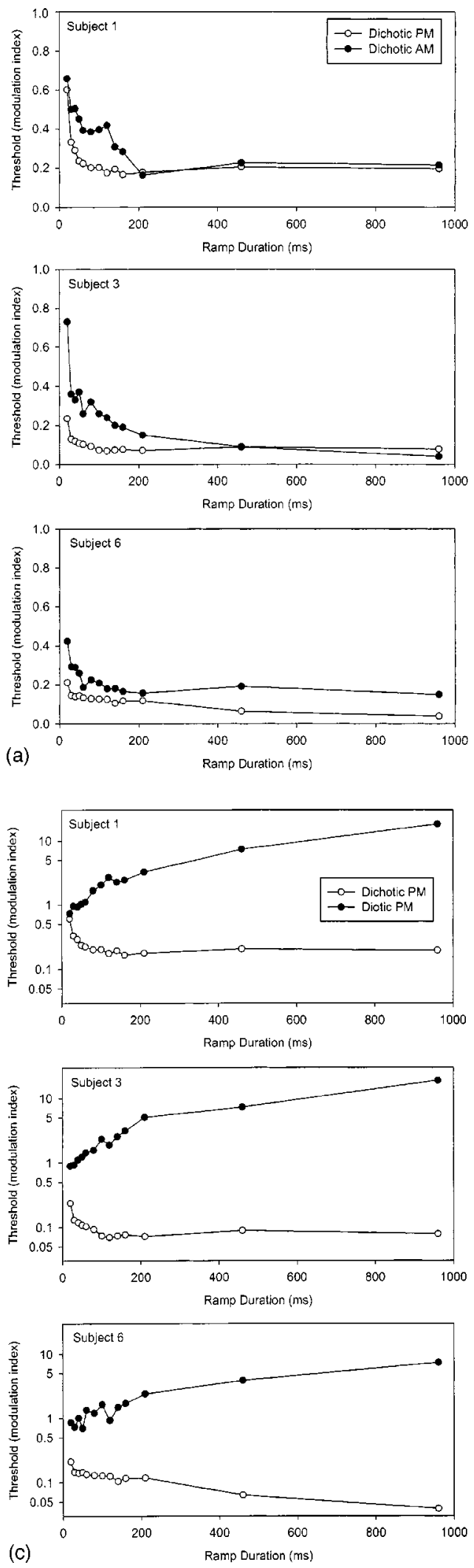

of the same order of magnitude (for the two subjects whose dichotic PM data could be fit with the curve), indicating that the effects of reducing ramp duration on sensitivity are comparable for each stimulus. Due to the presence of a dichotic advantage, subjects are making use of more than just diotic information when detecting dichotic PM at threshold. However, the dynamics of the mechanisms responsible for detect-
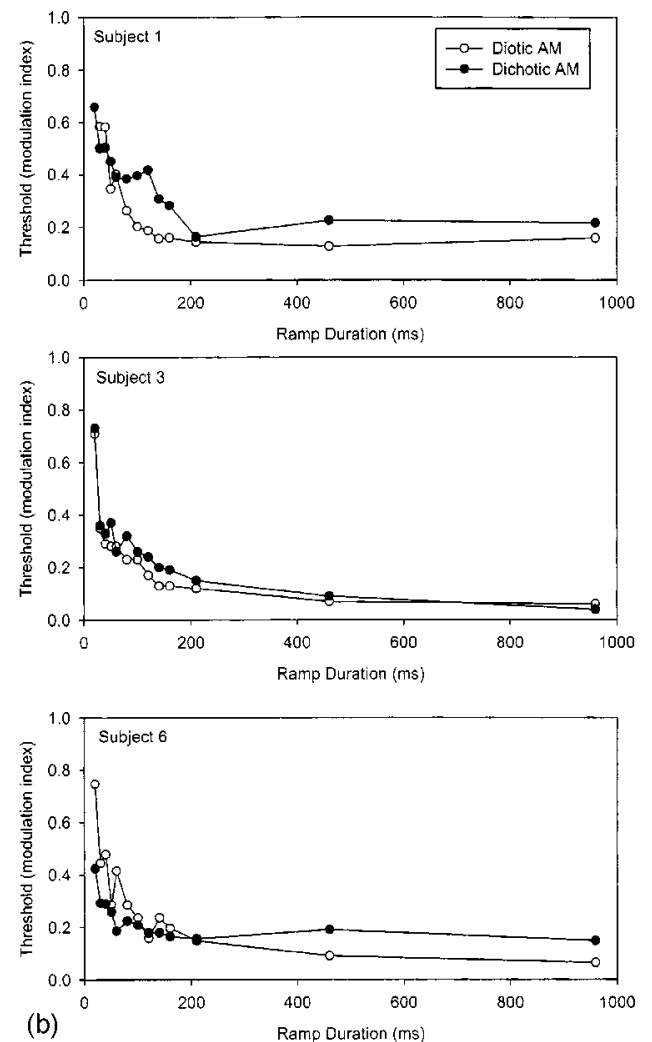

FIG. 4. Detection thresholds as a function of ramp duration: (a) thresholds for dichotic PM and AM; (b) thresholds for dichotic and diotic AM; and (c) thresholds for dichotic and diotic PM. Each graph in each figure shows data from a single subject. Note that the data in (c) are plotted on a logarithmic scale, in order to show the data more clearly.

ing dichotic PM and frequency pulses are approximately equally resistant to reductions in ramp duration. This observation might reflect the similar roles of temporal interval measurements, based on phase-locked neural impulses, which may underlie both frequency discrimination (Henning, 1970; Moore, 1972) and dichotic PM detection (Palmer et al., 1998). 


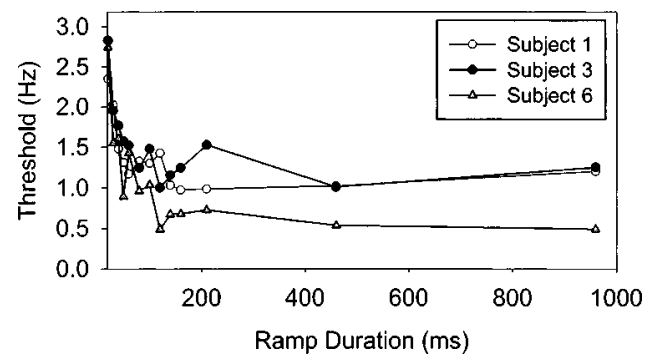

FIG. 5. Detection thresholds for diotic PM as a function of ramp duration, with thresholds converted to a measure of frequency pulse magnitude $(\mathrm{Hz})$ rather than phase (see text for details).

Time constants for dichotic and diotic AM are greater than those for dichotic PM and frequency pulse detection. There seems to be no simple pattern of relations between time constants for dichotic and diotic AM across subjects and there is quite a range of variability among them. Henning (1970) reported amplitude discrimination thresholds as a function of tone duration, and showed that sensitivity decreased with decreasing duration. However, the time-course of this decay in threshold was slightly faster than the equivalent decay for frequency discrimination thresholds, at least for low carrier frequencies. This observation is not in accordance with ours, that dichotic and diotic AM detection thresholds have a longer time constant than dichotic PM and frequency pulse thresholds. This difference may arise from differences between the effects of duration on amplitude discrimination and amplitude modulation detection: in the latter stimulus, the amplitude is only at its maximum for a brief time instead of for the whole duration of the tone. Grantham (1982, 1984) found that sensitivity to dynamic IIDs was more robust to temporal constraints than sensitivity to dynamic ITDs, a finding with which our data are also inconsistent. Grantham used broadband sounds and interaural differences were modulated in a sinusoidal manner, which could be the cause of the differences between our respective findings. With sinusoidal modulations, the number of times that the modulation depth reaches its maximum is increased as modulation rate increases, whereas our method of decreasing ramp duration does not have such an effect.

\section{Discussion of experiment 2}

\section{Comparisons with data from other studies}

The shape of the threshold-duration functions for our dichotic PM and AM stimuli is similar to that obtained by

TABLE II. Time constants in ms for dichotic PM and AM, diotic AM, and frequency pulses for three subjects, calculated using the method described in the text. No data are available for the dichotic PM data of subject 6 (see text for details).

\begin{tabular}{lcccc}
\hline \hline & Dichotic PM & Dichotic AM & Diotic AM & $\begin{array}{c}\text { Frequency } \\
\text { pulse }\end{array}$ \\
\hline Subject 1 & 11.7 & 82.4 & 38.8 & 18.8 \\
Subject 3 & 13.4 & 29.3 & 89.0 & 17.9 \\
Subject 6 & $\ldots$ & 86.6 & 81.0 & 24.7 \\
Mean (sd) & $12.6(1.2)$ & $66.1(31.9)$ & $69.6(27.0)$ & $20.5(3.7)$ \\
\hline \hline
\end{tabular}

TABLE III. Minimum integration times for detecting dichotic PM and AM, diotic AM and frequency pulses for three subjects, calculated using the method of Chandler and Grantham (1992). See text for details.

\begin{tabular}{lcccc}
\hline \hline & Dichotic PM & Dichotic AM & Diotic AM & $\begin{array}{c}\text { Frequency } \\
\text { pulse }\end{array}$ \\
\hline Subject 1 & 44.8 & 183.5 & 131.9 & 48.2 \\
Subject 3 & 46.8 & 92.0 & 292.7 & 48.6 \\
Subject 6 & - & 159.4 & 266.0 & 80.0 \\
\hline \hline
\end{tabular}

Grantham (1986) for movement simulated by stereophonic balancing of the sound in two speakers separated by $30^{\circ}$ (producing changing ITDs as well as changing ILDs). The time-course of the decay in sensitivity with decreasing duration appears to be slower in Grantham's data, since thresholds become elevated at durations longer than $200 \mathrm{~ms}$, although there are clear intersubject differences. One difference between the experimental paradigm employed by Grantham (1986) and the one employed here is that Grantham jittered the spatial position of stimulus onset, in an attempt to stop subjects from basing their decisions on the location of the onset or offset positions of either interval. This did appear to have a slight detrimental effect on the performance of some subjects and could account for some or all of the differences between our data. However, in a previous experiment where there was no jittering, thresholds still appeared to asymptote somewhere between 150 and $300 \mathrm{~ms}$ (Grantham, 1986).

The shape of the threshold-duration function for dichotic PM and dichotic AM (i.e., an exponential decay) is also similar to that calculated by Chandler and Grantham (1992) from their MAMAs at different velocities. However, the timecourse of this decay is faster in the present paper, both for dichotic PM and for dichotic AM. Chandler and Grantham (1992) used a free-field moving source with a carrier frequency of $500 \mathrm{~Hz}$. When detecting movement, subjects are therefore likely to have been making use of dichotic PM, rather than dichotic AM (as well as some spectral cues generated by the pinna and head), as a cue. This methodological difference could account for the differences between our findings.

Similarly, Chandler and Grantham's (1992) minimum integration times-about $300 \mathrm{~ms}$ for a $500-\mathrm{Hz}$ tone-are longer than the time constants reported here for either dichotic PM or dichotic AM. This difference probably results, in part, from differences in the method of calculating the integration time as well as differences in the data. Chandler and Grantham (1992) plotted MAMA against movement duration (similar to our plots in Fig. 4) and calculated integration time as the duration corresponding to a MAMA 25\% above the asymptote. For our data, we chose a different method to quantify time constants, by fitting similar curves with an exponential function. However, it is possible to calculate minimum integration times for our data in the same way as Chandler and Grantham (1992), based on our exponential fits: the value of $x$ [from Eq. (7)] when $y$ is $25 \%$ above the asymptote $y_{0}$.

Table III shows the minimum integration time for the 
data of subjects 1, 3, and 6, calculated using Chandler and Grantham's method. Although larger in value, the minimum integration times reflect a similar pattern of results to our time constants. Integration times are smallest for dichotic PM, around $44 \mathrm{~ms}$ for the two subjects whose data could be fit. They increase at least twofold for dichotic and diotic AM thresholds, but there is no observable pattern across these two stimuli. The minimum integration times reported here are clearly shorter than those reported by Chandler and Grantham (1992), probably as a result of methodological difference. However, the dissociation between integration times obtained for the different interaural modulations supports our hypothesis that there are significant differences between the processing of interaural phase and amplitude modulations.

\section{Sluggishness}

Recently, Bernstein et al. (2001) have used measurements of sensitivity to brief changes in ITD or IIDs under different conditions to model the temporal characteristics of sensitivity to these stimuli. They reported that sensitivity to both dynamic ITDs and IIDs is constrained by a single, symmetric, double-exponential, temporal window, characterized by a short $(0.09 \mathrm{~ms})$ and a longer $(13.8 \mathrm{~ms})$ time constant. The shortness of these time constants indicates that the binaural system may not be intrinsically sluggish. Nevertheless, the frequency response of this window had an initial lowpass segment, and it was found to reliably predict Grantham and Wightman's (1978) data for discrimination of dynamic ITDs.

In this study, it was found that thresholds for detecting dichotic PM are influenced by the duration of the modulation, and this is illustrated by their sharp increase when duration is reduced below about $150 \mathrm{~ms}$. However, the observation that duration has an even stronger limiting effect on thresholds for detecting dichotic and diotic AM, where sensitivity to dynamic interaural phase differences is not required for detection, suggests that dichotic PM detection, for tonal stimuli, might not be more sluggish than detection of other types of modulation. Other studies (e.g., Grantham, 1986; Chandler and Grantham, 1992) have found longer integration times for sound movement detection than were found for dichotic PM and AM detection in this study. It is therefore possible that although sound movement perception per se may be sluggish, perception of dichotic PM or AM of a $500-\mathrm{Hz}$ tone is not sluggish.

\section{SUMMARY AND CONCLUSIONS}

In summary, the findings presented in this paper suggest that there are significant differences between the psychophysically determined characteristics of dichotic PM and dichotic AM. These differences are reflected in comparisons of dichotic and diotic detection thresholds (dichotic advantage), in direction discrimination performance, and in the temporal limitations on detection.

\section{ACKNOWLEDGMENTS}

C.W. was supported by a studentship from the Medical Research Council.

${ }^{1}$ The reader might find it helpful to note that in our previous study (Witton et al., 2000), the degree of interaural phase modulation in the dichotic condition was always numerically identical to the degree of "monaural" phase modulation.

Bernstein, L. R., Trahiotis, C., Akeroyd, M. A., Hartung, K. (2001). "Sensitivity to brief changes of interaural time and interaural intensity," $\mathrm{J}$. Acoust. Soc. Am. 109, 1604-1615.

Carlile, S., and Best, V. (2002). "Discrimination of sound source velocity in human listeners," J. Acoust. Soc. Am. 111, 1026-1035.

Chandler, D. W., and Grantham, D. W. (1992). "Minimum audible movement angle in the horizontal plane as a function of stimulus frequency and bandwidth, source azimuth, and velocity," J. Acoust. Soc. Am. 91, 16241636

Grantham, D. W. (1982). "Detectability of time-varying interaural correlation in narrow-band noise stimuli," J. Acoust. Soc. Am. 72, 1178-1184.

Grantham, D. W. (1984). "Discrimination of dynamic interaural intensity differences," J. Acoust. Soc. Am. 76, 71-76.

Grantham, D. W. (1986). "Detection and discrimination of simulated motion of auditory targets in the horizontal plane," J. Acoust. Soc. Am. 79, 19391949.

Grantham, D. W., and Wightman, F. L. (1978). "Detectability of varying interaural temporal differences," J. Acoust. Soc. Am. 63, 511-523.

Green, G. G. R., Heffer, J. S., and Ross, D. A. (1976). "The detectability of apparent source movement effected by interaural phase modulation," J. Physiol. (London) 260, 49P.

Henning, G. B. (1970). "A comparison of the effects of signal duration on frequency and amplitude discrimination," in Frequency Analysis and Periodicity Detection in Hearing, edited by R. Plomp and G. F. Smoorenberg (Sijthoff, Leiden).

Henning, G. B., and Zwicker, E. (1984). "Binaural masking level differences with tonal maskers," Hear. Res. 16, 279-290.

König, E. (1957). "Effect of time on pitch discrimination thresholds under several psychophysical procedures; comparison with intensity discrimination thresholds," J. Acoust. Soc. Am. 29, 606-612.

Moore, B. C. J. (1972). "Frequency difference limens for short-duration tones," J. Acoust. Soc. Am. 54, 610-619.

Palmer, A. R., Jiang, D., and McAlpine, D. (1998). "Responses to sound motion: interaural amplitude and phase modulation in the inferior colliculus" in Psychophysical and Physiological Advances in Hearing, Proceedings of the 11th International Symposium on Hearing, edited by A. R. Plamer, A. Rees, A. Q. Summerfield, and R. Meedis (Whurr Publishers, London), pp. 368-375.

Perrott, D. R., and Musicant, A. D. (1977). "Minimum auditory movement angle: Binaural localization of moving sound sources," J. Acoust. Soc. Am. 62, 1463-1466.

Rayleigh, L. (1907). "On our perception of sound direction," Philos. Mag. 13, 214-232.

Turnbull, W. (1944). "Pitch discrimination as a function of tonal duration," J. Exp. Psychol. 34, 302-316.

Wichmann, F. A., and Hill, N. J. (2001a). "The psychometric function I: Fitting, sampling and goodness-of-fit," Percept. Psychophys. 63, 12931313.

Wichmann, F. A., and Hill, N. J. (2001b). "The psychometric function II: Bootstrap based confidence intervals and sampling," Percept. Psychophys. 63, 1314-1329.

Witton, C., Green, G. G. R., Rees, A., and Henning, G. B. (2000). "Monaural and binuaural detection of sinusoidal phase-modulation of a $500-\mathrm{Hz}$ tone," J. Acoust. Soc. Am. 108, 1826-1833.

Zwicker, E. (1952). "Die Grenzen der Hörbarkeit der Amplitudenmodulation und der Frequenzmodulation eines Tones" ("The limits of just perceptible amplitude modulation and frequency modulation of a tone"), Acustica 2, 125-133.

Zwicker, E., and Henning, G. B. (1985). "The four factors leading to binaural masking level differences," Hear. Res. 19, 29-47. 